\title{
BRCA1 and BRCA2: important differences with common interests
}

\author{
Rohini Roy, Jarin Chun and Simon N. Powell
}

The Opinion article we wrote (BRCA1 and BRCA2: different roles in a common pathway of genome protection. Nature Rev. Cancer 12, 68-78 (2012)) $)^{1}$ on the functions of BRCA1 and BRCA2 and their different roles in a common pathway of genome protection has generated correspondence by $\mathrm{Yu}$ and Shao (Initiation, evolution, phenotype and outcome of BRCA1 and BRCA2 mutation-associated breast cancer. Nature Rev. Cancer 24 Apr 2012 (doi:10.1038/ nrc3181-c1)) $)^{2}$ and by Joosse (BRCA1 and BRCA2: a common pathway of genome protection but different breast cancer subtypes. Nature Rev. Cancer 24 Apr 2012 (doi:10.1038/ nrc3181-c2) $)^{3}$. This correspondence implies that we have under-represented the differences between BRCA1 and BRCA2. On the contrary, as our title suggests, we emphasized two issues: the common pathway and the different roles. Given that the two proteins clearly have different biochemical functions and operate at different stages in the DNA damage response it is utterly remarkable that mutations in either gene produce an almost identical human syndrome, emphasizing the importance of their involvement in a common pathway. The differences in the subtypes of breast cancer seen in carriers of germline mutation in BRCA1 compared with carriers of $B R C A 2$ mutation are also fascinating and must be connected to their different roles. Our intention was not to minimize the differences between BRCA1 and BRCA2, but to see the entire picture more clearly. The Fanconi anaemia pathway works closely with homologous recombination (HR), but has an entirely different human syndrome that is inherited in an autosomal-recessive manner (rather than in an autosomal-dominant manner as is the case for hereditary breast and ovarian cancer). Hereditary breast and ovarian cancer and Fanconi anaemia are both diseases that derive from effects on proliferating cells, but their tissue specificity is entirely different.

Yu and Shao emphasize that timing is important in relation to how BRCA1 and BRCA2 mutations affect the breast cancer subtype. They argue that the differences observed in BRCA1- and BRCA2-mutation carriers must come late in the process of cancer development, after the initial onset of genomic instability. The reported observations about how often $B R C A 1$ and $B R C A 2$ behave as classic tumour suppressors, with the loss of the wild-type allele in tumour cells, are inconsistent ${ }^{4-9}$. Ensuring that wildtype $B R C A 1$ is absent in tumour cells and not present owing to contaminating normal tissues is always a challenge, especially in breast cancer, in which often considerably less than $50 \%$ of the cells in a specimen are tumour-derived. The idea that the influence of BRCA 1 and BRCA 2 mutations only occurs late in the process of carcinogenesis seems more difficult to support. Germline mutations usually indicate an early influence in most forms of hereditary breast cancer - it is not clear from Yu and Shao's correspondence just how the defective HR is occurring before the effect of BRCA1 and BRCA2 is observed. Yu and Shao also favour the model in which BRCA1 modifies the transcriptional profile of breast cancer cells: the presence of BRCA1 supports oestrogen receptor (ER) expression and represses the expression of cytokeratin 5 (CK5), CK17 and cadherin 3 $(\mathrm{CDH} 3)$. We agree that transcriptional alteration owing to the loss of BRCA1 function is a likely explanation for a number of the differences between BRCA1 mutation- and BRCA2 mutation-associated cancer. BRCA2 is probably fairly silent in transcription, which is why the profile of BRCA2 mutation-associated breast cancer is similar to that of non-hereditary breast cancer. Finally, the question of whether BRCA1 mutationversus BRCA2 mutation-associated cancer affects therapeutic outcome is questioned. If tumour size, nodal status and biomarker profile are accounted for, then the effect of being a mutation carrier is small.

Joosse also emphasizes that breast cancer subtypes are different in BRCA1- and BRCA2-mutation carriers, but that all mammary epithelial cells are derived from the mammary stem cell, which is ER-negative. He emphasizes that BRCA1 controls the development of ER-dependent luminal cells from progenitor cells, whereas BRCA2 has no such role. Therefore, luminal progenitor cells accumulate in contexts of BRCA1 deficiency, such as in a BRCA1-mutation carrier. Once the accumulation of luminal progenitors occurs, then the effect of HR deficiency produces genomic instability. In essence, this view reverses the more commonly accepted order in which carcinogenic events happen. However, the more we learn about models of carcinogenesis, the more we become convinced that it is the number of events that is important, not necessarily the order in which they occur ${ }^{10,11}$. The other assertion from Joosse is to question whether BRCA1 directly affects the expression of ER: he analysed 51 ER-positive luminal breast cancers, and seven (14\%) showed abnormalities on array-comparative genomic hybridization (aCGH), which indicates a BRCA1 deficiency ${ }^{12}$. Our own unpublished results agree with these observations (R.R., J.C. and S.N.P., unpublished observations). Six of the seven cases highlighted by Joosse showed BRCA1 mRNA downregulation, suggesting that BRCA1 expression does not affect ER expression. The debatable point here is whether the downregulation of BRCA1 is sufficient to cause the genetic instability that was detected by aCGH. We see substantial variation in the levels of BRCA1 mRNA and protein in sporadic breast cancer, but it does not correlate well with defective HR or with genomic instability detected by aCGH. In other words, there can be genomic instability in ER-positive breast cancer, which looks like BRCA1 deficiency, but that is not caused by a functional deficiency of the BRCA1 protein. The origin of this genomically unstable sporadic breast cancer (that is similar to BRCA1- or BRCA2-defective breast cancer) is the subject of ongoing research.

Rohini Roy, Jarin Chun and Simon N. Powell are at the Molecular Biology Program and Department of Radiation Oncology, Memorial Sloan-Kettering Cancer Center, 1275 York Avenue, New York, New York 10065, USA. Correspondence to S.N.P. e-mail:powells@mskcc.org doi: 10.1038/nrc3181-c3

Roy, R., Chun, J. \& Powell, S. N. BRCA1 and BRCA2 different roles in a common pathway of genome protection. Nature Rev. Cancer 12, 68-78 (2012).

Yu, K.-D. \& Shao, Z.-M. Initiation, evolution, phenotype and outcome of BRCA1 and BRCA2 mutation-associated breast cancer. Nature Rev. Cancer 24 Apr 2012 (doi:10.1038/nrc3181-c1). 3. Joosse, S.A. BRCA1 and BRCA2: a common pathway of genome protection but different breast cancer subtypes. Nature Rev. Cancer 24 Apr 2012 (doi:10.1038/nrc3181-c2).

4. Walsh, C. S. et al. Genome-wide loss of heterozygosity and uniparental disomy in BRCA1/2-associated ovarian carcinomas. Clin. Cancer Res. 14, 7645-7651 (2008).

5. King, T. A. et al. Heterogenic loss of the wild-type BRCA allele in human breast tumorigenesis. Ann Surg. Oncol. 14, 2510-2518 (2007).

6. Spearman, A. D. et al. Clinically applicable models to characterize BRCA1 and BRCA2 variants of uncertain significance. J. Clin. Oncol. 26, 5393-5400 (2008). Clarke, C. L. et al. Mapping loss of heterozygosity in normal human breast cells from BRCA $1 / 2$ carriers. $B r$. J. Cancer 95, 515-519 (2006).

8. Locke, I. et al. Loss of heterozygosity at the BRCA1 and BRCA2 loci detected in ductal lavage fluid from BRCA gene mutation carriers and controls. Cancer Epidemiol. Biomarkers Prev. 15, 1399-1402 (2006). 
CORRESPONDENCE

9. Brozek, l. et al. Loss of heterozygosity at BRCA $1 / 2$ loci in hereditary and sporadic ovarian cancers. J. Appl. Genet. 50, 379-384 (2009).

10. Vogelstein, B. \& Kinzler, K. W. Cancer genes and the pathways they control. Nature Med. 10, 789-799 (2004).

11. Parmigiani, G. et al. Design and analysis issues in genome-wide somatic mutation studies of cancer. Genomics. 93, 17-21 (2009).

12. Joosse, S. A. et al. Prediction of BRCA1-association in hereditary non-BRCA $1 / 2$ breast carcinomas with array-CGH. Breast Cancer Res. Treat. 116, 479-489 (2009).

Competing interests statement

The authors declare no competing financial interests. 